\title{
Semantic congruity effects in judgments of loudness
}

\author{
WILLIAM P. BANKS \\ Pomona College, Claremont, California 91711 \\ and \\ MARIA ROOT \\ Claremont Graduate School, Claremont, California 91711
}

\begin{abstract}
This research uses comparative judgments of the relative loudness of sounds to make a critical test of one theory of the mental representation of continuous physical attributes. The first two experiments find a semantic congruity effect, which is an interaction such that subjects can pick the louder of two loud sounds faster than the softer, and the softer of two quiet sounds faster than the louder. According to the theory under test, physical quantities are stored as points on a representational continuum, with a variance as well as a mean placement on it. The theory predicts the semantic congruity effect by assuming that the variance of placement of intensities on the representational continuum is a function of the direction of judgment: a soft sound will have less variance than a loud one when judged for softness and more when judged for loudness. Since the speed of making a judgment increases as variance decreases, the theory predicts a semantic congruity effect. However, for loudness, it can be shown that variance does not change in the manner assumed. The finding of a semantic congruity effect therefore disconfirms the theory. Alternative models are discussed.
\end{abstract}

This paper studies the mental processes that take place when people make comparative judgments of the loudness or softness of two sounds. Loudness was chosen for this study because, as a continuous psychophysical dimension, it offers a means for testing models of the mental representation of continuous attributes. These models fall into two general categories. The first is the analog model, in which the mental representation is continuous and in which the mental processing is performed with continuous quantities. The analog medium may be an abstract unidimensional representation, as is the case with the models of Jamieson and Petrusic (1975) and Marks (1972), or it may be a concrete, image-like medium of internalized perception, as in the models suggested by Moyer (1973) and Paivio (1975). Banks (1977) has termed these, respectively, analog continuum and imagery models. Both models assert that the memory representation is analog and that the computation of the correct response is performed by use of this ana$\log$ representation. These fundamental similarities suffice to put the two sorts of theory in the same category despite major differences in other respects.

The other general sort of model is usually termed the discrete model. It apparently acquired this term

This research was supported by NSF Grant BNS 78-17442. Requests for reprints should be sent to W.P.B. at the Department of Psychology, Pomona College, Claremont, California 91711. through opposition to the analog models, but since the only existing discrete models assume a semantic coding or categorical labeling process, it seems more appropriate to term them "semantic" rather than "discrete." The term "discrete" implies a more general class of models that has not been developed. Semantic models assume that processing, and sometimes storage, of information is in terms of categorical tags or semantic features. These happen to encode attributes discretely, but their primary attribute is that they carry meaning in the way natural language concepts do. Banks (1977) has reviewed the applications of semantic models to predicting reaction time (RT) in comparative judgment and showed that they can account for the effects generally cited as evidence for analog models and predict a number of other effects as well. Anderson (1978) has recently come to the conclusion probably shared by many that analog and semantic models vary only in the ease with which they can explain various phenomena, but as yet no phenomenon has been found that decisively rejects one or the other modeling approach.

One phenomenon that has been offered as decisive between the classes of model is the semantic congruity effect. This effect is an interaction between the instructions (e.g., "choose smaller" vs. "choose larger") and the region of the dimension or continuum under test. Two small stimuli, for example, might take longer to discriminate under "choose 
larger" than "choose smaller" instructions, and the reverse would be true in this example for discrimination among two large stimuli. This example describes a crossover interaction, but a crossover is not required to show a semantic congruity effect. All that is needed is an interaction of any form such that the instructions lead to a faster mean processing time when applied to stimuli located at the end of the dimension they name than at the opposite end.

The semantic coding model predicts the congruity effect by assuming that the stimuli are given codes before an attempt is made to choose the correct response, and it is these codes that are used in the selection of a response. The codes given to the stimuli are determined in large part by the region of the continuum from which the stimuli come. If both stimuli are large, for example, they are likely to be both coded as "large." The semantic congruity interaction emerges because the choice is made more quickly when the stimulus code matches the terms of the instruction than when it does not. Thus, the instructions "choose larger" will be executed more quickly when stimuli are encoded "large" than when they are encoded as "small"; the converse is true for the instructions "choose smaller," and the semantic congruity effect is predicted. Banks (1977), Banks and Flora (1976), and Banks, Fujii, and Kayra-Stuart (1976) discuss the semantic coding model's predictions concerning comparative judgments in more detail, for other effects as well as for the semantic congruity effect.

Analog models have advanced three sorts of explanations of the semantic congruity effect. We will term these expectancy, search, and end-anchoring mechanisms. The expectancy effect assumes that the instructions, coming before the stimuli are presented, can "set" the subject either appropriately or inappropriately to process the stimuli. The semantic congruity effect is predicted because the instructions set the subject to process stimuli near the pole specified by the instructions, and stimuli near the pole are assumed to be processed faster than those from the opposite end of the continuum. Banks and Flora (1977) rejected the expectancy hypothesis because they still got a semantic congruity effect when they presented the instructions $2 \mathrm{sec}$ after the stimuli. Moyer and Dumais (1978) suggest, however, that subjects defer processing while waiting for the instructions. We find this suggestion implausible because Banks and Flora's subjects were considerably faster overall with post- than with prestimulus instructions, but we are nevertheless currently conducting further experiments on the role of expectancy, which is not the topic of the present paper.

The second mechanism, search, was proposed by Moyer and Bayer (1975). They postulated that presentation of a symbolic stimulus triggered a search through memory for a representation of it. The search is assumed generally to begin at the end of the continuum specified by the instructions. The representation is therefore found quicker, and the response is made faster, when the stimuli are near the end of the continuum specified by the instructions than when they are at the opposite end, and the semantic congruity effect is predicted. Banks (1977) brought up two lines of evidence against the search model. The first is that virtually all experiments requiring comparative judgments of serial position in a list yielded the fastest RTs for items at both ends, with slower responses in the middle, no matter what the instructions. The search model should predict a monotonic increase in RT as pairs more remote from the pole of the instructions are tested. The second line of evidence is the existence of the congruity effect with poststimulus instructions. Because the search for the representation of the stimuli should be complete by the time the instructions are presented, the instructions cannot influence the search in the manner proposed.

The remaining mechanism of the three assumes an anchor effect in the decision process. This mechanism requires an analog continuum model of the representation. Jamieson and Petrusic (1975) and Marks (1972) have different versions of the anchor effect, but both assume that mental operations on analog quantities derived from the stored analog continuum are needed to compute the answer (it could be said that the analog continuum is a sort of "mental slide rule" in these models). Jamieson and Petrusic assume that the analog measure of the magnitude of a given stimulus is the distance of its representation from the pole of the continuum specified by the instructions. The mechanism for computing the response is not specified, but it is assumed that the time required for making the decision is inversely proportional to $R$, the ratio of the two analog magnitudes. The semantic congruity effect is predicted because $R$ will be larger for a given stimulus pair when the instructions specify the pole near the instructions than when they specify the opposite pole. Unfortunately, the model predicts a monotonic increase in RT as pairs a constant distance apart are located progressively farther from the pole of the instructions. This model thus has the same problem as the search model because the position effects for ordered series are rarely monotonic. Banks (1977) discusses this and other problems of the Jamieson and Petrusic model in more detail.

It seems that the only remaining mechanism by which an analog model might predict the semantic congruity effect is that of Marks. The present paper tests this mechanism. Marks' model is similar to Jamieson and Petrusic's in that the items to be discriminated among in comparative judgment are assumed to be mapped to points on an internal representational continuum. It also assumes, in common 
with their model, that in retrieving the information for use in processing, subjects take the distance of the representational point from one of the two anchor points as the measure of the magnitude of the stimulus. The difference between the models is that Marks' mechanism works by assuming that the variance of the measure increases as a function of the mean distance of the representation from the anchor used for retrieving the information. Reaction time is predicted by the principle that the greater the variance of two points separated by a constant distance, the longer it should take to discriminate them. To avoid confusion we will, from now on, refer to Marks' model as a discriminal dispersion model, rather than an end-anchor model, of which it is but one version.

The discriminal dispersion model of Marks predicts the semantic congruity effect by assuming that the instructions specify the pole of the continuum from which the measurement is made. If the stimuli to be judged are at the end of the continuum specified by the instructions, the variance of the dispersions about the mean representations of the stimuli will be less than if the stimuli are at the opposite end of the continuum. Thus, the RTs will be faster when the instructions and the stimuli are from the same end of the continuum than when they are from opposite ends of the continuum, and the semantic congruity effect is predicted.

Marks' model has, to our knowledge, been tested only by Banks, Clark, and Lucy (1975). They showed that the semantic congruity effect for judgments on an artifical continuum was additive with changes in RT caused by stimulus discriminability. The conclusion they drew was that whatever process is responsible for the semantic congruity effect is separate from the process that discriminates the stimuli. They argued for a multistage chain of processing in opposition to Marks' single stage in which perceptual identification yields the effects on RT of both stimulus discriminability and stimulus-instruction congruity. The Banks et al. model has a first stage that assigns codes to the stimuli (and whose duration is affected by stimulus discriminability), followed by a second stage that attempts to select the code specified by the instructions (and whose duration is affected by the congruity between stimulus codes and the instructions).

While the Banks et al. study disconfirms Marks' use of the discriminal dispersion model to account for semantic congruity and discriminability effects in a single stage, it does not provide an empirical test of the discriminal dispersion mechanism itself. The possibility remains that Marks' model could be saved as the second stage of a two-stage process. The present experiment attempts a direct test of the discriminal dispersion model of the semantic congruity effect. The strategy of this experiment was to see if the semantic congruity effect is obtained for an attri- bute for which the discriminal dispersions for judgments are known not to vary with the pole of judgment. The research of Schneider and Lane (1963) on loudness seems to demonstrate conclusively that the discriminal dispersion of the loudness of sounds does not vary with pole of judgment. Schneider and Lane's subject set an attenuator to produce the soundpressure level subjectively equivalent to levels of softness or loudness designated numerically by the experimenter. The variance of produced sound levels varied only with the sound-pressure level of the sound and not at all with the direction of judgment on the continuum. The productions provide an estimate of the mean placement of and variance of the mental representation of a given quantity on either the loudness or softness continuum. It would seem, then, that the discriminal dispersion model would predict no semantic congruity effect for this continuum. If a semantic congruity effect is found for loudness judgments, the discriminal dispersion model would be rejected, and the analog models would be left without a means to predict the semantic congruity effect.

\section{EXPERIMENT 1}

\section{Method}

On each of 288 trials, the eight subjects heard over headphones a sound of a different loudness in each ear and, while timed, pressed a microswitch on the side of the louder on a random half of the trials and the softer on the other half. Instructions to indicate the louder or softer were varied from trial to trial and were given before each trial. Both sounds were $1,000-\mathrm{Hz}$ sine waves, switched on by an analog gate that created no audible switching transients. One-half second after a trial was initiated, the apparatus would present the first sound for $700 \mathrm{msec}$, then the other for $700 \mathrm{msec}$, with no silent interval or overlap between them. The timer began measuring the RT with the onset of the second stimulus. The subjects were informed that they should respond as quickly as they could and still maintain near-perfect accuracy, and that they should not wait until the end of the second stimulus before responding.

Stimuli were presented at three different mean levels of intensity, termed "quiet," "'medium," and "loud." Two pairs of stimuli were used at each of the three levels to facilitate counterbalancing and to make it difficult for subjects to guess the correct answer in a sequentially presented pair. The sound-pressure levels reported below for the stimuli are based on mesurements taken from the headphones with a Realistic sound-level meter, where $0 \mathrm{~dB}$ is .0002 dynes $/ \mathrm{cm}^{2}$. The absolute sound-pressure levels are only approximate because of the difficulty of obtaining readings from headphones that accurately reflect the sound-pressure levels delivered to the ear of a subject. The relative decibel levels are, however, accurate, since they are based on the voltages presented to the headphones (within the range used in the experiment, the earphones are linear, according to the sound-pressure-level meter). The two quiet pairs, by our measurements, were $59 \mathrm{~dB} / 65 \mathrm{~dB}$ and $65 \mathrm{~dB} / 77 \mathrm{~dB}$, the two medium pairs were $69 \mathrm{~dB} / 75 \mathrm{~dB}$ and $75 \mathrm{~dB} / 87 \mathrm{~dB}$, and the two loud pairs were $79 \mathrm{~dB} / 85 \mathrm{~dB}$ and $85 \mathrm{~dB} / 97 \mathrm{~dB}$. As can be seen, the three ranges are spaced $10 \mathrm{~dB}$ apart and the spread between stimuli within the ranges causes some overlap between the medium pairs and pairs from the quiet and loud sets. The overall levels were chosen by the authors to span the range from quiet to loud as nearly as possible. The amount of spread between members of a pair was based on pilot 
work in which an attempt was made to make the stimuli discriminable enough that subjects would never make an error. Because the stimuli were presented for only $700 \mathrm{msec}$ (and were never simultaneously present), the seemingly large differences between the intensities within a pair still did not make it easy for all subjects to discriminate the members of the pair.

The requirement that pairs be discriminable enough to preclude errors of perception resulted in a very conservative test of the semantic congruity effect. The wide span within pairs and the overlap between pairs kept them from being as clearly loud or soft as we would have liked; thus, the likelihood that they would be coded as "soft" or "loud" and so yield a semantic congruity effect was reduced. Furthermore, we could not use tones that sounded very soft because soft tones could not be reliably heard in $700 \mathrm{msec}$ or in any reasonably short presentation interval. Consequently, our range was restricted such that the "quiet" pairs were actually in a subjectively middling range of loudness, and the "loud" ones were subjectively quite loud (but far short of painfully loud). It would seem that, with the restricted range of stimuli and the blurring of distinctions between levels because of overlap in intensities of members of pairs in the three ranges, if a semantic congruity showed itself it would have to be a robust effect indeed.

Stimuli were generated by a Hewlett-Packard Model 650A oscillator, which also set the overall level of attenuation in 10-dB steps. The output of the Hewlett-Packard was connected to a specially designed box that contained timing and switching circuits, the attenuators used to determine the relative loudness of the two stimuli on a given trial, and amplifiers to drive the headphones.

The subjects were divided into two counterbalancing groups. One of these groups heard the first of the two stimuli on the right ear for the first half of the session and then heard the first one on the left for the second half, while the other group started off hearing the first member of each pair on the left ear, then heard the first on the right. The side of correct response, the intensities, and the instructions were completely randomized within each half session for both groups of subjects.

The subjects were eight graduate and undergraduate students of the Claremont Colleges, paid $\$ 2$ for the approximate $50 \mathrm{~min}$ required for the session. An additional subject was paid but rejected from the experiment because of a high error rate. No hearing test was used to select subjects, since poor performance in the task itself would serve to select out subjects with poor acuity for intensity differences or with unilateral hearing loss (and a consequent systematic misperception of relative intensities, leading to a high error rate).

\section{Results}

The congruity interaction in RT was obtained, but it fell short of significance, with an $F(2,12)$ of 1.5 , $p>, 10$. However, there was a reliable interaction between the congruity effect and the order of presentation, $F(2,12)=6.31, p<.025$. This interaction was such that when the first stimulus was the one the subject should choose, a strong congruity effect was obtained, but when the second one was the correct choice, there was none (in fact, it went slightly in the wrong direction in the latter case). Separate analyses of variance for the two cases confirm this conclusion. When the first stimulus was correct, the congruity effect yielded an $F(2,12)$ of $6.24, p<.025$, but the $F$ ratio was only .69 when the second stimulus was correct. Figure 1 shows the RTs for the two instructions and three intensity levels, plotted separately for the first-ear-correct and second-ear-correct conditions. It is apparent that a robust congruity effect (of the fun-
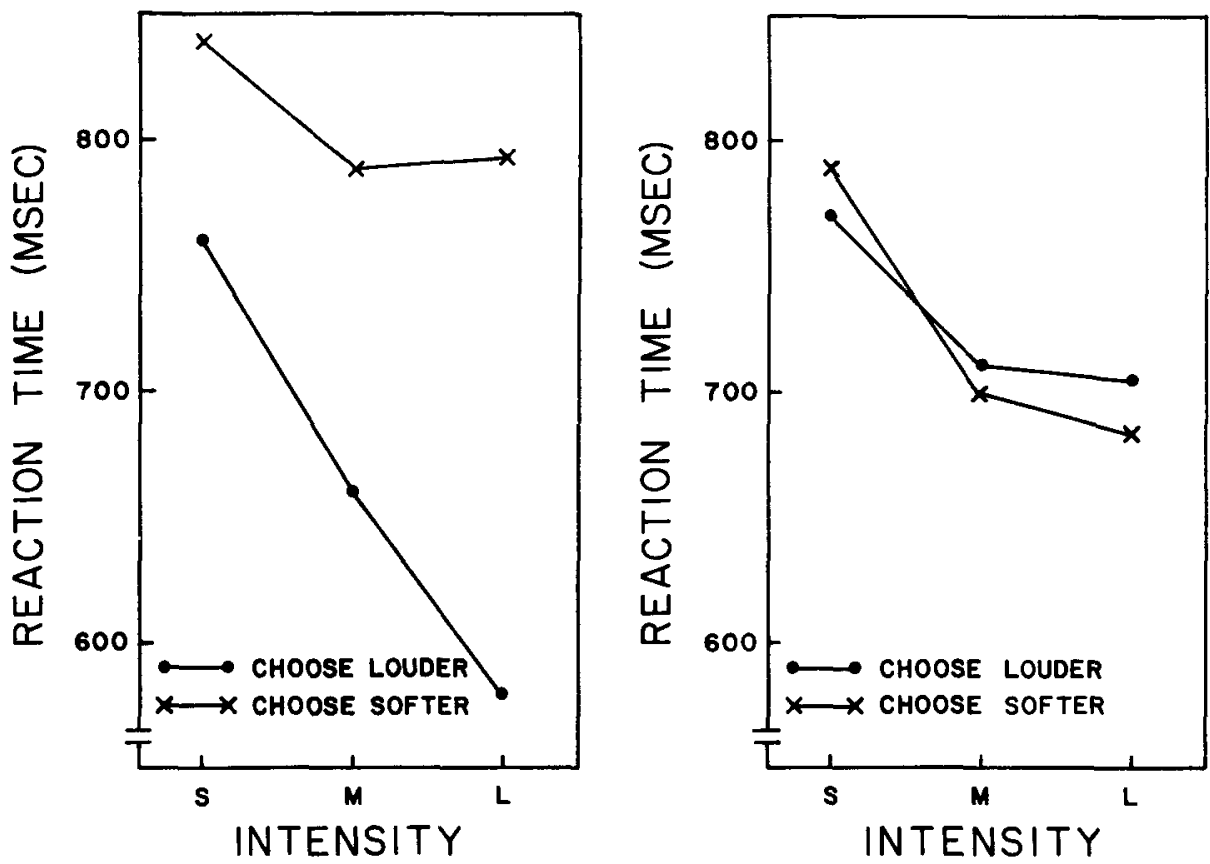

Figure 1. Reaction time to pick either the louder or the softer of two 1,000-Hz sequentially presented tones, plotted for soft (S), medium (M), and loud (L) intensities, as defined in the text. Left panel plots results when the first of the two tones is correctly chosen, and the right panel plots the results when the second one is chosen. 
nel form) is found for the first stimulus, and a negligible interaction for the second.

It seems reasonable to suppose that the funnel form of the congruity effect came from the fact that extremely quiet stimuli were not used but very loud ones were. By this supposition, the quiet stimuli may not always have been coded as quiet but the loud ones were nearly always coded as loud. Thus, no difference in processing time for the instructions was found for the quiet stimuli, but the loud stimuli, being usually coded as "loud," were processed faster for the "choose louder" than the "choose quieter" instructions.

The error rates were low, ranging from $.1 \%$ to $8 \%$ across subjects, with a mean of $\mathbf{4 . 2 \%}$. The RTs and errors were positively correlated with $r=.76$ over conditions. The difference between the congruity effects for the first- and second-stimulus-correct conditions cannot be ascribed to a speed-accuracy tradeoff because error rates showed a pattern very similar to the RTs within these two conditions. The error rates plotted in the same way as the RTs in Figure 1 show a strong congruity effect when the first stimulus is correct. There were a few more errors for second than for first stimulus correct ( $3.5 \%$ vs. $5.5 \%)$ ).

The finding of a congruity effect for the first but not the second of two sequentially presented stimuli is exactly the opposite of what has been found previously for comparative judgments with sequentially presented pairs. Two comparative judgment experiments have presented the pair of stimuli successively and studied the congruity effect. The first of these was an experiment of Wallis and Audley (1964) in which judgments of relative pitch were made, and the second was reported by Banks (1977, pp. 143-145) and used a purely symbolic continuum, size of object, symbolized by words. Both of these gave a congruity effect only for the second stimulus. Banks (1977) suggested that the similar pattern of results may indicate that a common mechanism of judgment is involved. The present finding of a congruity effect only with the first stimulus shows that the pattern is not general. However, there may be special circumstances in this case that caused this particular outcome. For example, the instructions in this case may have differed in some way from the instructions in the others and changed the subjects' strategy of responding while the second stimulus was present. Also, the subjects may have responded to the change in loudness from the first to second stimulus rather than to the two individual levels, whereas the change itself may not have been as perceptually salient in Wallis and Audley's and Banks' experiments.

We decided that the best solution to the various problems of interpretation posed by the results of Experiment 1 was to find a way to present the two sounds simultaneously. Pilot work showed that a pair of $1,000-\mathrm{Hz}$ tones could not be used if presented simultaneously because they were not heard as a pair, but were fused into a single tone whose location in space was determined by the relative sound-pressure levels of the two tones. The relative judgment, once the subject was practiced, became a judgment of apparent spatial position rather than of softness or loudness. We found that a $500-\mathrm{Hz}$ sine wave and a square wave with a fundamental frequency of $2,000 \mathrm{~Hz}$ would not fuse when presented simultaneously. Subjects heard them as two separate sounds, localized on opposite sides of the head, under all of our conditions of simultaneous dichotic presentation at all sound-pressure levels. These two stimuli were used for relative judgments in Experiment 2.

\section{EXPERIMENT 2}

\section{Method}

The method and procedure were identical to those of Experiment 1 , with the following exceptions. The stimuli, a $500-\mathrm{Hz}$ sine wave and a square wave with a fundamental of $2,000 \mathrm{~Hz}$, were presented simultaneously for $700 \mathrm{msec}$ at only two different mean intensity levels, loud and quiet.

Four pairs of sine- and square-wave stimuli were used within each intensity level. Specified in terms of decibels of sound-pressure level at the earphone measured in Experiment 1, with the intensity for the square wave specified first in each pair, they were, for the loud level: $87 \mathrm{~dB} / 101 \mathrm{~dB}, 114 \mathrm{~dB} / 101 \mathrm{~dB}, 104 \mathrm{~dB} / 84 \mathrm{~dB}$, and $104 \mathrm{~dB} / 111 \mathrm{~dB}$. For the quiet tones, the intensities were $67 \mathrm{~dB} /$ $81 \mathrm{~dB}, 94 \mathrm{~dB} / 81 \mathrm{~dB}, 84 \mathrm{~dB} / 64 \mathrm{~dB}$, and $84 \mathrm{~dB} / 91 \mathrm{~dB}$. These intensities were selected to sound appropriately loud or soft and to create approximately equally discriminable pairs. The overlap between two of the stimuli used in soft and loud pairs was scarcely noticeable because the other stimulus in each of the pairs affected by the overlap gave the pair as a whole the appropriate loud or soft sound. However, as in Experiment 1, we could not use extremely quiet stimuli and keep either a low error rate or a brief stimulus duration. The test for a congruity effect is, consequently, somewhat conservative, as it was in Experiment 1.

All variables were fully randomized within each subject's block of trials, but one half of the subjects always had the sine wave on the left ear and the square wave on the right, and the other half had them reversed. Subjects contributed 8 RTs to each of the 16 different stimulus conditions, or 32 to each of the 4 main conditions plotted in Figure 2. Fourteen subjects from the same pool as used in Experiment 1, paid the same, participated in this experiment.

\section{Results}

The semantic congruity effect is shown in Figure 2. This interaction is reliable, with an $F(1,13)$ of 5.5 , $p<.05$. As can be seen, the effect is quite large in terms of mean RT. Eleven of the 14 subjects showed a semantic congruity effect of the form seen here $(p<.03)$. The funnel form of the effect is to be expected from the fact that very loud stimuli were used but not very quiet ones.

Errors were very infrequent $(1.8 \%$ overall) and tended to fall in the conditions that had long RTs. The correlation between RT and error rate for the four main conditions is $\mathbf{. 6 5}$. Because of the very low 


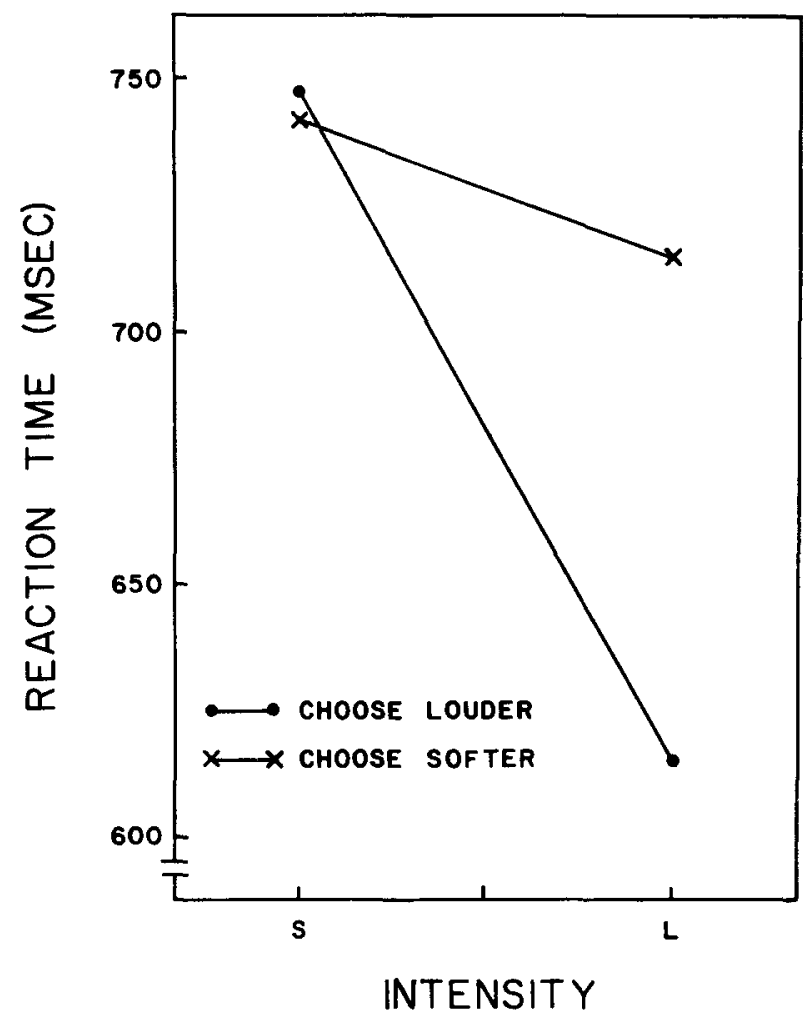

Figure 2. Reaction time to pick the louder or softer of two simultaneously presented tones, plotted for soft (S) and loud (L) intensities, as defined in the text.

error rate, further analysis of errors has little meaning.

\section{EXPERIMENT 3}

The theoretical importance of the congruity effect for comparative judgments of loudness and softness depends on the assumption that the variance of the internal representation of sound intensities is the same whether the representation encodes subjective loudness or softness. The Schneider and Lane (1963) study supports the proposition that the variance does not differ for softness and loudness, but there are a number of objections that could reasonably be made to the application of the Schneider and Lane findings to the present situation. Most important of these is the fact that they used one very experienced subject repeatedly, while we used naive subjects in a single session with minimal training. It is possible that both a congruity effect and a difference between variances of judged softness and loudness are characteristic of early stages of practice, which we studied, but no difference in variances and no congruity effect are characteristic of later stages of practice, which Schneider and Lane studied. To test whether both the variance effects and the congruity effect vary with practice, it would be necessary at least either to test highly practiced subjects for the congruity effect or to determine whether naive subjects have the same variability of representation for loudness and softness. As it is, we have found a congruity effect for naive subjects and rely on data from a well-trained subject for estimates of variability of the continuum. We chose in this experiment to examine the variability of loudness and softness for relatively naive subjects.

In Experiment 3, we used subjects who had either served in Experiment 1 or were used in related pilot work, in both cases having had the experience sometime between 2 and 6 months prior to this experiment. We thus used subjects who had only a minimal amount of experience making judgments of loudness and softness, but whose results in the judgment task would certainly be appropriate to compare with the comparative judgment results. The technique used to assess the variability of the mental representation of loudness was magnitude production. Subjects were given control of an attenuator and were asked to produce loudnesses or softnesses of a magnitude specified numerically as a ratio to a standard magnitude.

\section{Method}

Apparatus and Stimuli. The same equipment used for Experiments 1 and 2 was employed here, with a ten-turn potentiometer inserted in the line. The potentiometer allowed the subject to vary the sound-pressure level from about $120 \mathrm{~dB}$ (no attenuation) to about $45 \mathrm{~dB}$. (The same technique for measuring sound pressure in decibels used for Experiments 1 and 2 was used here.) Over its range, the attenuator was very nearly logarithmic in its action; a constant angular turn of the knob produced a constant amount of change in decibels of attenuation. The attenuator was mounted in a hand-held box, and the subjects were instructed not to look at the knob while setting intensities. The stimulus was the $500-\mathrm{Hz}$ sine wave used in Experiment 2, presented through the earphones to the right ear only on all trials.

Procedure. The experimenter read magnitude production instructions identical to Schneider and Lane's to each subject. The same sound-pressure level $(85 \mathrm{~dB})$ was used as the standard for both softness and loudness productions, and the subjects were told that its magnitude was 10 on either the softness or loudness continuum, whichever was being judged. Subjects judged softness and loudness in two separate blocks. Half the subjects had the softness block first and half had the loudness block first. The standard was presented at the beginning of each block, and as often thereafter as the subject requested it. Within each block, each subject made 25 productions of either softness or loudness. The quantities produced were to correspond to subjective magnitudes of $2,5,10,20$, or 40 . (These were defined as having a subjective softness or loudness of $.2, .5,1.0,2.0$, or 4.0 , respectively, times that of the standard.) The subjects made five productions of each of these subjective magnitudes in each block in a randomized order.

At the beginning of each block, the experimenter presented the standard to the subject and read the instructions. At the beginning of each trial, the subject was told the subjective magnitude for the next production, and the stimulus was turned on until the subject was satisfied he or she had set the specified magnitude. The voltage delivered to the earphones was then recorded, the stimulus was turned off, and the quantity for the next production was read to the subject. When the stimulus was turned on again for the next setting, it was always at the level of the previous setting. 


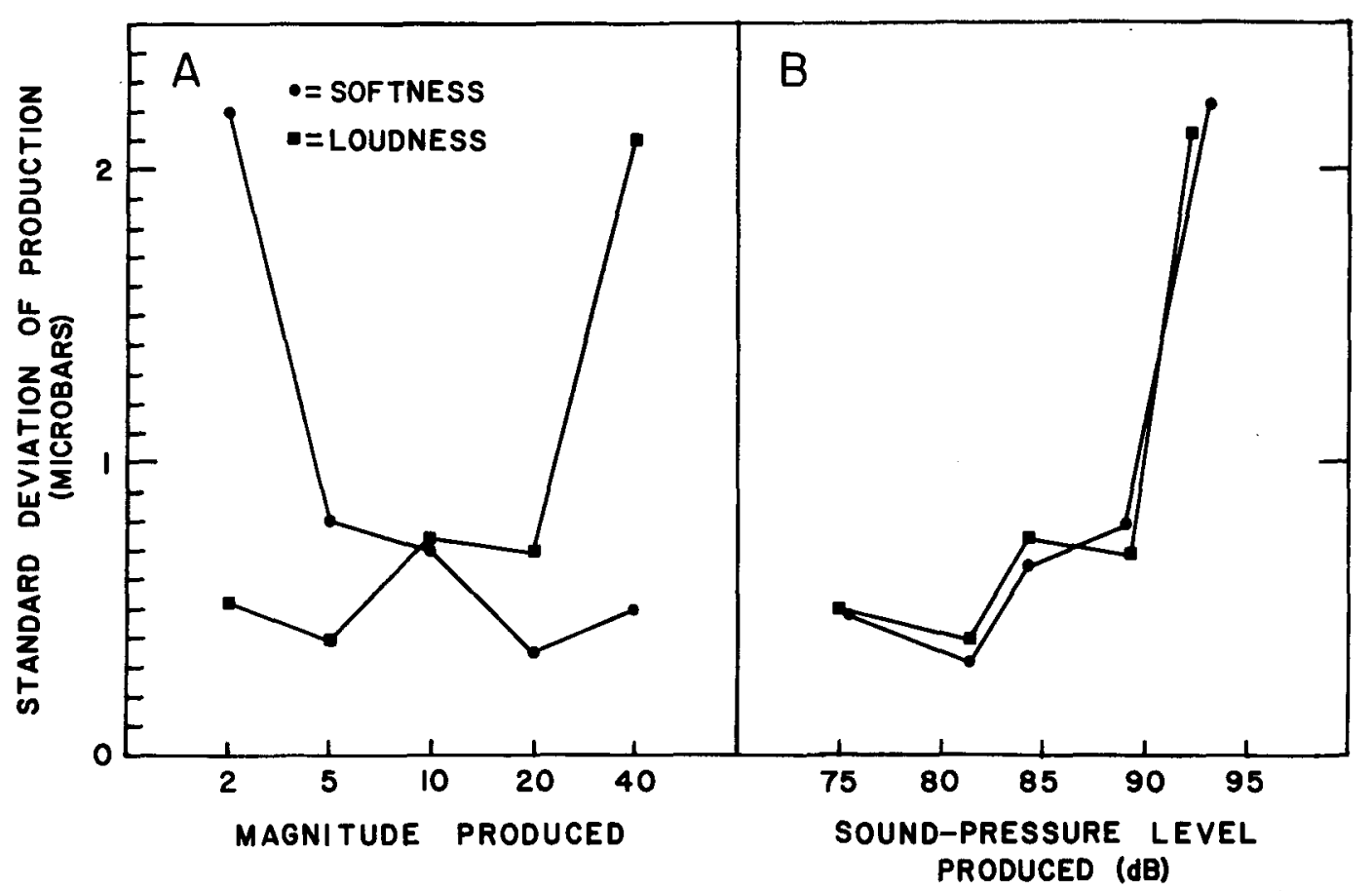

Figure 3. (A) Standard deviation of loudness and softness productions (in microbars, where 1 microbar equals .002 dynes $/ \mathrm{cm}^{2}$ ) plotted as a function of subjective magnitude of softness or loudness; standard deviation is not predicted by subjective magnitude or direction of judgment. (B) Standard deviation (in microbars) of loudness and softness productions plotted as a function of sound-pressure level; standard deviation is well predicted by mean level of physical stimulus, with virtually no influence of direction of judgment.

Subjects. Six students in the Claremont Colleges, aged 20-28 years, volunteered to serve without pay for the approximate $30 \mathrm{~min}$ the experiment required. All had previously served in one, and only one, experiment on comparative judgment of loudness using this apparatus.

\section{Results and Discussion}

Schneider and Lane's findings were substantially replicated. Figure 3 shows the standard deviation of the settings in microbars, plotted in two different ways to illustrate the fact that variability is determined by the physical intensity rather than the distance from the zero-point on the subjective continuum. In Figure $3 \mathrm{~A}$, the standard deviation of the production is plotted as a function of the specified degree of softness or loudness to be produced. As can be seen, the function is quite different for softness and loudness. The variability of loudness increases as subjective loudness increases, and the variability of softness decreases as softness increases. Variability of the setting does not increase with distance from the anchor point or zero point of the subjective continuum. The interaction seen in Figure $3 \mathrm{~A}$ is reliable, with an $F(4,20)$ of $7.65, p<.001$. On the other hand, Figure $3 \mathrm{~B}$ shows that the variability of the production is well predicted by the sound-pressure level and that the direction of the subjective scale (softness or loudness) has little effect. The main effect of sound-pressure level in Figure 3B has an $F(4,20)$ of $7.69, p<.001$, and the interaction testing the difference between the functions of sound-pressure level for softness and loudness has an $F<1.0$. The main effect of instructions also has an $F<1.0$. In sum, the variability of the setting is a function of the stimulus intensity, with apparently no systematic influence of subjective anchor point.

One possibility that might be considered is that subjects convert instructions for both softness and loudness productions to values on a common loudness scale. If they did, then the variances of loudness and softness settings might be expected to increase in a similar way with sound-pressure level. Several considerations detract from the plausibility of a translation. First, the productions of subjects who had softness instructions first are indistinguishable from those of subjects who had loudness instructions first. If there is a translation from softness to loudness, it would have to be the result of a preexperimental bias and be relatively uninfluenced by instructions. It seems unlikely that subjects would universally take the strategy of translating softness to loudness before making a production. Second, in both this study and Schneider and Lane's, the variance functions for softness and loudness were almost identical. A translation from softness to loudness would insert an additional mapping for softness productions that 
does not exist for loudness and would add a component of variance to softness productions and not to loudness. Softness productions at any given soundpressure level should therefore have more variance than the comparable loudness productions. Since they do not, and in fact the variances are approximately equal for softness and loudness, a translation in either direction seems unlikely.

\section{GENERAL DISCUSSION}

The main finding of a semantic congruity effect for a continuum in which the variance is the same for judgments relative to both anchor points (i.e., for judgments of loudness and softness) implies that the discriminal dispersion model cannot account for the effect. This model is therefore rejected for comparative judgments of loudness, but we are not interested only in loudness. Can we conclude that the model is not valid in general? We point out that it seems unreasonable to maintain the discriminal dispersion model for only those continua that do show the necessary relation between direction of judgment and variance of representation and reject it for continua that do not. It is better to find a model that can handle both cases. Although the discriminal dispersion model is not eliminated for cases that may show the necessary relation between variance and direction of judgment, considerations of parsimony should cause us to eliminate the discriminal dispersion model until critical evidence against the competing models that can now handle all cases is found. At present, there is no such evidence, nor in fact have any continua been identified for which the relation between variance and direction of judgment needed by the discriminal dispersion exists. Marks (1972) did not measure any discriminal dispersions in his paper on the model.

The version of the semantic coding model used by Banks et al. (1976) for comparative judgments of the relative numerical magnitudes of digits seems applicable to the present situation. In that model, the stimulus is assumed to be mapped onto an internal analog representational continuum much like that assumed by Marks (1972), but the response is not computed directly with analog quantities on the continuum. Rather, codes are generated for the stimuli on the basis of their positions on the continuum. The code-generation process uses a criterion to decide on the code for a stimulus. Only two codes are used, and the assigned code is determined by whether a stimulus falls above or below the criterion. In this case, a sound will be classified as either "loud" (meaning "louder than the criterion" and symbolized L +) or "soft" ("softer than the criterion," or S+). The response is made by matching a stimulus code with the correct response code specified by the instructions (also symbolized as $\mathrm{L}+$ or $\mathrm{S}+$ ).
The decline in RT as stimuli are separated more on the continuum results from the fact that the further the distance they are apart, the more likely the two are to receive different codes because they are more likely to straddle the criterion. If they receive different codes, one of them will match the instruction, and a response can be made rapidly. If they are both on the same side of the criterion, they will receive the same codes (both $\mathrm{L}+$ or $\mathrm{S}+$ ), and further processing will be necessary to distinguish them. The further processing is assumed to change $\mathrm{L}+/ \mathrm{L}+$ to $\mathrm{L}+/ \mathrm{L}$ and $\mathrm{S}+/ \mathrm{S}+$ to $\mathrm{S}+/ \mathrm{S}$. Because this further processing adds to the RT, and because the two are more likely to fall on the same side of the criterion as they are moved closer together on the continuum, the RT will increase as the distance decreases.

The semantic congruity effect comes about because stimuli are generally coded in terms of the region of the continuum on which they fall. If both are loud, for example, they are likely both to fall above the criterion and to be initially encoded as $\mathrm{L}+/ \mathrm{L}+$ and eventually as $\mathrm{L}+/ \mathrm{L}$. A match to the instructions $\mathrm{L}+$ can be made quickly, but to decide which will match $\mathrm{S}+$ requires a further transformation, adding to the RT. Likewise, a stimulus pair at the soft end of the continuum will take longer under the instructions $\mathrm{L}+$ than $\mathrm{S}+$, and the congruity effect is predicted.

We have recently considered an alternative to the semantic coding model that would also use semantic or categorical codes to predict the semantic congruity effect. This alternative model would rely on a form of competition among processing codes much like Stroop interference. Rather than resulting from processes of code manipulation in a set of serial processing stages as assumed in the semantic coding model, the congruity effect would come from interference between conflicting codes generated in parallel. We will refer to this as the semantic interference model. In the present task, by this model, the loudness of the loud stimuli would be an irrelevant attribute that gives rise to an implicit code "loud" that could interfere with processing of the "choose quieter" instructions and facilitate the "choose louder" instructions. The opposite pattern of interference and facilitation would occur for the quiet stimuli, and the semantic congruity effect would be predicted. The interference in the classical Stroop task is probably with the response (Dyer, 1973), but it may be with other processes in the present task. Clark and Brownell (1975), for example, found evidence for operation of Strooplike interference with generation of perceptual codes in a perceptual comparative judgment task.

Although the present experiments are not designed to distinguish between the semantic interference and semantic coding models, we think it is important to mention the interference model so that tests of it may be considered by others as well as by us. At present, 
we can think of no effects that give one model a clear advantage over the other. For example, both models seem to handle variations in the size of the congruity effect equally well, but they do it by different means. The semantic coding model assumes a constant duration (for a given set of material) for the congruity-ensuring transformation of codes that leads to the semantic congruity effect, and the various factors that influence the magnitude of the effect operate by varying the probability of performing the congruity transformation rather than by influencing its duration (Banks et al., 1976, have an explicit mathematical treatment of these assumptions).

The interference model has at least two mechanisms it could use to predict variations in the congruity effect. The first would assume that the degree of interference was a consequence of the degree of relationship between the interfering attribute and the required response. The more similar they are, the more interference or facilitation, and the larger the congruity effect. Such a similarity effect would be analogous to the pattern found in Stroop color-word interference whereby interference with color naming is greater when the interfering word names a color (and is therefore similar to the response) than when it does not. The interference model might also assume that the relative speed of processing of the relevant and interfering attributes determines the size of the congruity effect. In the Stroop effect, the faster to be processed attribute interferes more with the slower to be processed than the slower with the faster (Banks \& Barber, 1977; Dyer, 1973), and naming a color is slower than reading a word. Thus, in the Stroop task there is little interference with reading the word, no matter what the color of ink, but a conflicting word interferes with naming the ink color.

In comparative judgment tasks, by analogy, the size of the congruity effect would be a function of the relative speed of processing of the criterial and interfering attributes. If the interfering attribute were processed faster than the criterial attribute, there would be a larger congruity effect than if it were processed more slowly. It is possible that the interference model, by assuming relative speed differences among the criterial and interfering attributes, can explain many processing effects in addition to variations in the size of the semantic congruity effect. For example, Clark and Brownell's finding that the interfering attribute affected perceptual code generation while Banks et al. (1975) found it affected a decision or responseselection process may be a result of the differing speeds of processing interfering attributes in the two cases. The interfering attribute that is processed quickly should conflict with events early in processing (such as perception), and one that is processed slowly should conflict with events late in processing (such as the response). It remains to be seen whether the semantic interference model will have more explanatory power than the semantic coding model. The apparent flexibility of the model may evaporate when all of its assumptions are made explicit and subjected to test.

One question of some importance is that of whether the magnitude productions of subjects measure their mental representation of sound intensity. An alternative technique to magnitude production for investigating the mental representation would be magnitude estimation. According to this technique, subjects provide numbers expressing their judgments of the degree of softness or loudness of sounds. If we had used magnitude estimation in this study rather than magnitude production, we probably would have found the variance of the estimate to increase as the numbers subjects used got larger. Thus, the results would probably have shown the variance of the magnitude estimations to increase with sound-pressure level for loudness judgments and to decrease with sound-pressure level for softness judgments. The problem with interpreting such a finding is that the variance effects could come from the characteristics of the numerical response scale rather than the internal representation of sound intensity. Using magnitude estimation to get scales of softness and loudness causes the size of the numbers used at each end of the continuum to be perfectly confounded with the direction of judgment. It would be impossible to tell whether variance increases with sound-pressure level for loudness and decreases for softness because of the different anchor points used or because in both cases estimates using larger numbers have more variance. Magnitude productions are not subject to this confounding. Whether producing a loudness or a softness, the subject is always setting a sound-pressure level and the response scale itself does not vary with instructions the way it would with magnitude estimation. However, if there is a difference in the variability of the internal representation of sound intensity depending upon whether it is internally constructed as softness or loudness, then the difference should exhibit itself in the way in which subjects produce magnitudes of softness or loudness. Since productions do not show a change in variance with direction of judgment, we can conclude that the mental representation of the continuum does not. It therefore seems that there is no way for the discriminal dispersion mechanism to predict the semantic congruity effect.

\section{REFERENCES}

Anderson, J. R. Arguments concerning representations for mental imagery. Psychological Review, 1978, 85, 249-277.

BANKS, W. P. Encoding and processing of symbolic information in comparative judgments. In G. H. Bower (Ed.), The psychol- 
ogy of learning and motivation (Vol. 11). New York: Academic Press, 1977.

Banks, W. P., \& Barber, G. Color information in iconic memory. Psychological Review, 1977, 84, 536-546.

Banks, W. P., Clark, H. H., \& Lucy, P. The locus of the semantic congruity effect in comparative judgments. Journal of Experimental Psychology: Human Perception and Performance, $1975,1,35-47$.

Banks, W. P., \& Flora, J. Semantic and perceptual processes in symbolic comparisons. Journal of Experimental Psychoiogy: Human Perception and Performance, 1977, 3, 278-290.

Banks, W. P., Fujil, M., \& Kayra-Stuart, F. Semantic congruity effects in comparative judgments of magnitude of digits. Journal of Experimental Psychology: Human Perception and Performance, 1976, 2, 435-447.

Clark, H. H., \& Brownell, H. H. Judging up and down. Journal of Experimental Psychology: Human Perception and Performance, 1975, 104, 339-352.

Dyer, F. N. The Stroop phenomenon and its use in the study of perceptual, cognitive, and response processes. Memory \& Cognition, 1973, 1, 106-120.

Jamieson, D. G., \& Pérrusic, W. M. Relational judgments with remembered stimuli. Perception \& Psychophysics, 1975, 18, 373-378.
Marks, D. F. Relative judgment: A phenomenon and a theory. Perception \& Psychophysics, 1972, 11, 156-160.

MOYER, R. S. Comparing objects in memory: Evidence suggesting an internal psychophysics. Perception \& Psychophysics, 1973, 13, 180-184.

Moyer, R. S., \& BAyer, R. H. Mental comparison and the symbolic distance effect. Cognitive Psychology, 1976, 8, 228-246.

Moye R, R. M., \& Dumais, S. T. Mental comparison. In G. H. Bower (Ed.), The psychology of learning and motivation (Vol. 12). New York: Academic Press, 1978.

Paivio, A. Perceptual comparisons through the mind's eye. Memory \& Cognition, 1975, 3, 635-647.

Schneider, R., \& Lane, H. Ratio scales, category scales, and variability in the production of loudness and softness. Journal of the Acoustical Society of America, 1963, 35, 1953-1961.

WAllis, C. P., \& Audley, R. J. Response instructions and the speed of relative judgments: II. Pitch discrimination. Britisit Journal of Psychology, 1964, 55, 121-132.

(Received for publication February 27, 1979; revision accepted June 5, 1979.) 